Mary Harmony B. Que, MD, MBA'

Maria Rina T. Reyes-Quintos, MD, PhD ${ }^{1,2,3,4}$

'Department of Otolaryngology

Head and Neck Surgery

The Medical City

${ }^{2}$ Hearing and Dizziness Center

The Medical City

${ }^{3}$ Department of Otorhinolaryngology

College of Medicine - Philippine General Hospital

University of the Philippines Manila

${ }^{4}$ Philippine National Ear Institute

National Institutes of Health

University of the Philippines Manila
Correspondence: Dr. Maria Rina T. Reyes-Quintos

Department of Otorhinolaryngology

Ward 10, Philippine General Hospital

Taft Ave., Ermita, Manila 1000

Philippines

Phone: (632) 526-4360

Fax: (632) 525-5444

Email: rinatrq@yahoo.com

The authors declared that this represents original material that is not being considered for publication or has not yet been published or accepted for publication elsewhere, in full or in part, in print or electronic media; that the manuscript has been read and approved by all the authors, that the requirements for authorship have been met by each author, and that each author believes that the manuscript represents honest work.

The authors signed disclosures that there are no financial or other (including personal) relationships, intellectual passion, political or religious beliefs, and institutional affiliations that might lead to conflict of interest.

Presented at the Philippine Society of Otolaryngology - Head and Neck Surgery Descriptive Research Contest (1st place). August 10, 2017. Natrapharm, The Patriot Bldg., Parañaque City.

\section{Evaluation of the Newborn Hearing Screening Program in The Medical City Based on Joint Commission on Infant Hearing (JCIH) 2007 Position Statement Quality Indicators}

\begin{abstract}
Objective: The objective of this study is to evaluate the newborn hearing screening program in The Medical City based on the Joint Committee on Infant Hearing (JClH) 2007 Position Statement Quality Indicators.
\end{abstract}

\section{Methods:}

$\begin{array}{ll}\text { Design: } & \text { Cross - Sectional Survey } \\ \text { Setting: } & \text { Tertiary Private Hospital } \\ \text { Participants: } & \text { All newborns who underwent newborn hearing screening in The }\end{array}$
Medical City for the year 2015.

Results: Of 2,010 patients delivered in the hospital in year 2015, 1,986 (98.8\%) were screened. Among the 59 babies with initial "refer" results, 15 (25.42\%) "referred" a second time while 24 $(40.68 \%)$ "passed" the rescreening. Twenty (33.89\%) did not undergo rescreening (10 were classified as dropouts while another 10 did not undergo rescreening for various reasons. Of those who "referred" during rescreening, only $9(60 \%)$ had further evaluation done with ABR/ASSR. Among these, 4 (26.66\%) had hearing loss and proceeded with the appropriate monitoring and management while $5(33.33 \%)$ had normal hearing.

Conclusion: The current newborn hearing screening program in the Medical City was able to reach $\mathrm{JClH} 2007$ quality indicators for screening but not for confirmation of hearing loss. All patients with hearing loss were managed with early rehabilitation.

Keywords: Newborn hearing screening, otoacoustic emission test, auditory brainstem response test, auditory steady state response test

Along with other countries around the globe, the Philippines has established newborn hearing screening programs to detect hearing loss in children at a very young age. Screening is only the beginning of the audiological testing battery which often involves rescreening and additional audiological tests to confirm or repudiate the initial findings before diagnosis and rehabilitation.' The Joint Committee on Infant Hearing $(\mathrm{JClH})$ is an association made up of members who are 


\section{ORIGINAL ARTICLES}

experts in their fields of audiology, otolaryngology, pediatrics and nursing. They are responsible for making recommendations concerning the early identification of children with or at risk for hearing loss. In 2007, they published the JCIH 2007 Position Statement which includes the benchmarks and quality indicators as a guide and goal for hospitals with hearing screening programs. ${ }^{2}$

The Medical City is a tertiary private hospital with a newborn hearing screening program. The Medical City is also a Joint Commission International $(\mathrm{JCl})$ accredited institution. This means that it is committed to quality improvement and dedicated to providing the best possible care for their patients. Thus, the impetus for this study - which is to improve the newborn hearing screening program of the hospital. A study in the United States of America from year 2005-2011 showed a rescreen follow up rate of $65 \%$ in $2011,{ }^{3}$ similar to findings in an unpublished Medical City study of $66.8 \%$ in $2009 .{ }^{4}$ According to $\mathrm{JCIH}$, the quality indicators for screening include a screening rate of $>95 \%$, and a "refer" rate of $\leq 4 \%{ }^{2}$ For the quality indicators for confirmation of hearing loss, $90 \%$ must complete a comprehensive audiological evaluation. ${ }^{2}$ To the best of our knowledge, there has also been no published data about JCI and $\mathrm{JClH}$ nor $\mathrm{JCl}$ and newborn hearing screening programs.

It is important to know how The Medical City newborn hearing screening program compares with the $\mathrm{JClH}$ Quality Indicators for Screening and Confirmation of Hearing Loss. This information can tell us if the program is reaching the goals set by the quality indicators, and if not, the possible reasons and solutions. The objective of this study is to evaluate the newborn hearing screening program in The Medical City based on the Joint Committee on Infant Hearing (JClH) 2007 Position Statement Quality Indicators.

\section{METHODS}

With Institutional Review Board approval, this cross - sectional study retrieved the records of all newborns delivered in 2015 in Medical City, a tertiary private hospital in the Philippines. The total number of babies who underwent newborn hearing screening and the number of babies who obtained "pass" and "refer" results using an Otoport Lite TEOAE Model (Otodynamics Ltd., UK) were gathered and recorded from the logbooks accomplished by the nurses after testing. As a routine hospital practice before discharge, babies with persistent "refer" results on hearing screening are automatically scheduled for rescreening at the Hearing and Dizziness Center of The Medical City after 1 month. They are given reminders of their rescreening schedule by the Hearing and Dizziness Center (HDC) staff and the pediatric resident rotator via phone calls. The HDC staff routinely inquire about the reasons why the mother (or caregiver) refused or cancelled their appointment. The parents answers are recorded in logbooks. These were the logbooks searched by the investigators.

The investigators searched for the rescreening records of those who had "refer" results and these were also collated. The parents of the babies with "refer" results had all been advised to undergo rescreening at the Hearing and Dizziness Center as well as diagnostic testing using $A B R$ and ASSR if the result was still a "refer." The MADSEN AccuScreen ${ }^{\circledR}$ two-step (OAE/ABR) hand-held screening system (GN Otometrics, $A / S$, Denmark) had been used for rescreening, and the Navigator Pro Multiple Auditory Steady-State Evoked Response System II (Bio-logic ${ }^{\circledR}$ MASTER $^{\circledast}$ II) (Natus, CA, USA) for additional diagnostic testing.

Parents or caregivers of all babies who deviated from the protocol anywhere in the course of the program were identified. Those who failed to follow-up for rescreening were contacted by non-recorded phone calls and asked through open-ended questions why they did not undergo the recommended tests. Parents or caregivers of babies who were identified to have hearing loss based on diagnostic testing were questioned if they had already proceeded with rehabilitation and, if applicable, why not. The number of attempts to contact respondents was also recorded. Those who could not be contacted by the Hearing and Dizziness staff after 3 attempts, and for whom no evidence of a repeat test in the Medical City could be found, were classified as dropouts.

Based on the responses, factors such as 'lack of education / knowledge about the need for a repeat test,' lack of time,' unavailable testing center (scheduling/ machine problems), 'financial constraints,' 'poor customer service,'advise not to proceed with retesting,',opted to transfer to another hospital or testing center,'have forgotten or do not care for a repeat test,'changed doctors,' were identified and recorded.

Percentages were used to determine the fraction of newborn babies screened, the fraction of babies who had a "pass" or a "refer result," fraction of babies returning for rescreening and those who did not, fraction of babies who had a "pass" or a "refer" result after the repeat screen, fraction of babies who underwent confirmatory tests and those who did not. The software used was Microsoft Excel for Windows Version 16.0.6769.2017 (Microsoft Corp., Redmond, WA, USA).

\section{RESULTS}

Out of 2,010 babies delivered in our hospital for 2015, 1,986 (98.8\%) were screened. The remaining 21 patients (1\%) were either discharged against medical advice, transferred or expired before the hearing tests could be done while $3(0.1 \%)$ refused testing.

Of the 1,986 babies screened, 1,927 (97.02\%) had initial screening test results of "pass" and 59 (2.97\%) had initial screening test results of "refer." This is seen in the first column in Figure 1. 


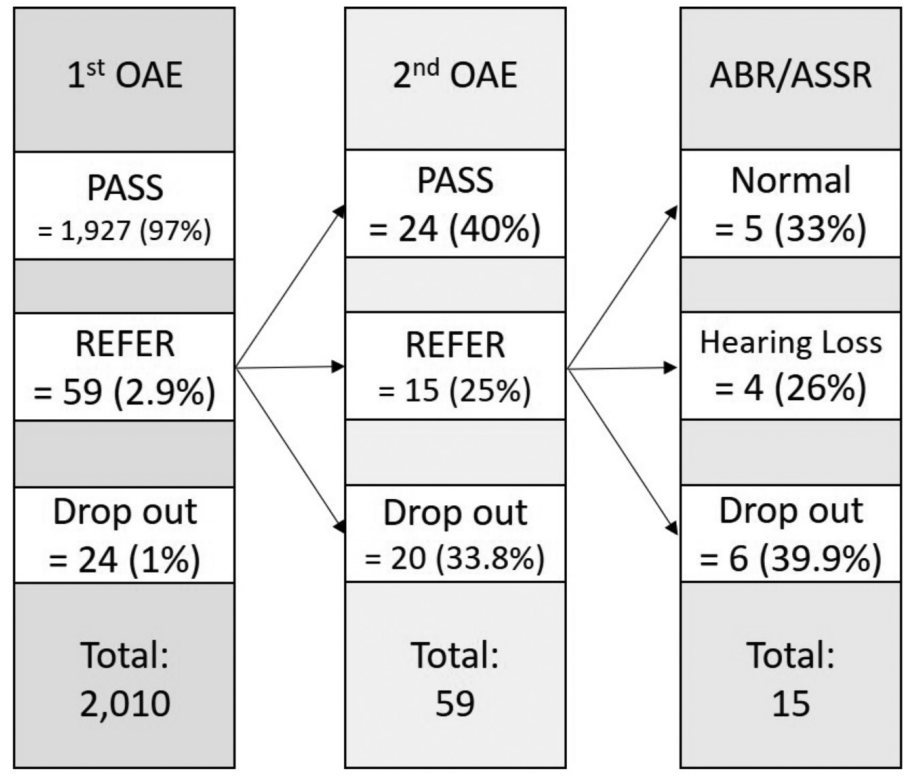

Figure 1. Number of patients with a "pass" or "refer" and number of drop outs on 1st and 2nd OAE and number of patients with normal or abnormal results after ABR/ASSR tests.

Among the 59 babies with initial "refer" results, 15 (25.42\%) "referred"a second time while $24(40.68 \%)$ "passed" the rescreening. (Figure 1) Twenty (33.89\%) did not undergo rescreening, 10 of these were classified as dropouts while another 10 did not undergo rescreening for other reasons seen in Table 1 below.

Table 1. Reasons why parents did not bring their child for rescreening

\begin{tabular}{|l|c|}
\hline Reason for not having a repeat hearing screening & $\begin{array}{l}\text { Number of } \\
\text { Patients }\end{array}$ \\
\hline Parent has no time to bring patient for repeat OAE & 2 \\
\hline Patient had other more pressing medical problems & 1 \\
\hline Parent advised by pediatrician to observe patient & 3 \\
\hline $\begin{array}{l}\text { Parent advised to have repeat hearing test but has not yet } \\
\text { been able to do so }\end{array}$ & 1 \\
\hline $\begin{array}{l}\text { OAE machine was broken and parent couldn't schedule a } \\
\text { repeat OAE }\end{array}$ & 2 \\
\hline $\begin{array}{l}\text { Parent clinically assessed patient to have no problems in } \\
\text { hearing hence did not schedule a repeat OAE }\end{array}$ & 1 \\
\hline Total & $\mathbf{1 0}$ \\
\hline
\end{tabular}

Of the 15 babies with rescreening results of "refer," 9 (60\%) had further evaluation with ABR/ASSR. Of these, 4 (26.66\%) were diagnosed to have hearing loss and 5 (33.33\%) had normal hearing. Five other babies (33.33\%) were considered dropouts, and 1 (6.66\%) had not undergone $A B R / A S S R$ because the mother had no time to bring the infant for the test. (Figure 1)
Among those with hearing loss, 2 (50\%) had unilateral mild hearing loss for which they were advised close monitoring, 1 (25\%) had bilateral hearing loss (right moderate hearing loss and left moderate to severe hearing loss) for which hearing aids were prescribed, while 1 (25\%) had bilateral profound hearing loss for which preparation for cochlear implantation is being started. Hence, only $0.2 \%$ of 1,986 patients screened had hearing loss and all have proceeded with the appropriate management.

Our results show that The Medical City was able to reach the $\mathrm{JCIH}$ 2007 quality indicators for screening but not the quality indicators for confirmation of hearing loss. (Table 2)

Table 2. JCIH Quality Indicators for Screening and Confirmation of Hearing Loss compared with The Medical City results

\begin{tabular}{|l|c|c|}
\hline Quality Indicators for screening & $\begin{array}{c}\text { JCIH 2007 Quality } \\
\text { Indicators }\end{array}$ & $\begin{array}{c}\text { The Medical } \\
\text { City }\end{array}$ \\
\% Babies Screened & $\geq 95 \%$ & $98.8 \%$ \\
$\%$ Babies who"refer" & $<4 \%$ & $0.75 \%$ \\
\hline $\begin{array}{l}\text { Quality Indicators for Confirmation of } \\
\text { Hearing Loss }\end{array}$ & $\geq 90 \%$ & $60 \%$ \\
\% Diagnostic testing completed & & \\
\hline
\end{tabular}

\section{DISCUSSION}

The Medical City was able to reach the $\mathrm{JCIH} 2007$ quality indicators for screening but not for confirmation of hearing loss.

This problem has also been seen in other places besides the Philippines and successful interventions have been done for better compliance with the many diagnostic audiological procedures needed prior to starting rehabilitation. A University of Illinois study showed that they were able to decrease loss to follow-up and documentation rate from $71.36 \%$ to $46.36 \% .^{5}$ Their strategy involved educating the primary health care providers and parents and preparing English and Spanish materials about the importance of the program. Program implementors also met regularly to monitor their progress. ${ }^{5}$ Two studies in Massachusetts showed a loss to follow-up rate of 25\% and $50.9 \%$ respectively. ${ }^{6,7}$ These losses to follow-up were attributed to several factors such as being non-white, receiving public insurance and a mother who smoked during pregnancy. Those who had unilateral and mild to moderate hearing loss were also more likely to be lost to followup. ${ }^{6,7}$ A possible solution to this problem was proposed by Hunter in 2016. ${ }^{8}$ Her research showed that collaborating with the Women, Infants, and Children program in Ohio (wherein staff members would contact family members to remind them of their pending schedules based on 
their newborn hearing screening protocol), as well as counselling and educating them, had a strong impact on decreasing the loss to follow up rate from $33 \%$ to $9.6 \% .^{8}$ These same strategies may also be applied in our setting: making sure that stakeholders are regularly reminded and updated about the existence and importance of the newborn hearing screening program, hiring dedicated staff responsible for advising the parents after screening (who should also be monitored so that they impart the proper information in the proper manner), and writing easy to understand brochures in the vernacular, may be instrumental in educating reminding and encouraging the parents to continue with the newborn hearing screening program and that it is worthwhile to do so.

One of the limitations of this study is the study population; only patients who were born in The Medical City on the year 2015 were included in this study and these may not represent the larger population of Filipino children with hearing loss since majority of the mothers who give birth in the said institution are among the higher classes who have better access to proper healthcare. The methodology can also be improved to include a uniform script of questioning with a set of choices for the parents to select as reasons why they did not come back for further testing. Future studies can take these into consideration.

This study showed that The Medical City has attained the $\mathrm{JClH}$ benchmarks for screening but still needs to improve with regards to follow up for confirmation of hearing loss which was $60 \%$ compared to a minimum of $90 \%$ benchmark from $\mathrm{JClH}$. Steps should be taken to address this low follow up for confirmation of hearing loss so that the hearing screening program in The Medical City will fulfill its goal of early screening and intervention for children with hearing loss.
1. Universal Newborn Hearing Screening and Intervention Act of 2009. Republic Act No. 9709. Republic of the Philippines. (Aug 12 2009).
2. Joint Committee on Infant Hearing. Year 2007 position statement: Principles and guidelines for early hearing detection and intervention programs. Pediatrics. 2007 Oct; 120(4): 898-921. DOI: 10.1542/peds.2007-2333; PubMed PMID: 17908777.
3. Alam S, Gaffney M, Eichwald J. Improved Newborn Hearing Screening Follow-up Results in More Infants Identified. J Public Health Manag Pract. 2014 Mar; 20(2): 220-223. DOI: 10.1097/ PHH.0b013e31829d7b57. PubMed PMID: 23803975 PubMed Central PMCID: PMC4470168.
4. Abratique RJ, Batayola MC, Reyes-Quintos MRT. Current state of the universal new born hearing screening program at the Medical City. Unpublished 2009: 1-17.
5. Reducing Loss to Follow-up after Failure to Pass Newborn Hearing Screening. Illinois Program Narrative. Chicago: The University of Illinois. 2010-2011. 1-18. [cited 2017 June 12] Available from:https://www.infanthearing.org/stategrants/grants_supplemental_2009/4-IL\%20 Supplemental\%202010-2011.pdf.
6. Liu CL, Farrell J, MacNeil JR, Stone S, Barfield W. Evaluating loss to follow-up in newborn hearing screening in Massachusetts. Pediatrics. 2008 Feb; 121(2) e335-43, doi:10.1542/peds.2006-3540. Epub 2008 Jan 10. PubMed PMID:18187812.
7. Crouch E, Probst J, Bennett $\mathrm{K}$ and Carroll T. Evaluating loss to follow-up in newborn hearing screening in a southern state. JEHDI. 2017; 2(1):40-47. [cited 2017 June 12] Retrieved from http://digitalcommons.usu.edu/cgi/viewcontent.cgi?article=10438\&content=jehdi.
8. Hunter LL, Meinzen-Deir, Wiley S, et al. Influence of the WIC program on loss to follow- up for newborn hearing screening. Pediatrics. 2016 Jul; 138(1) e20154301. PubMed PMID: 27307144 PubMed Central PMCID: PMC4925076.

